\title{
Dietary health of medical workers: who's taking care of it?
}

\section{Wei Chen}

Department of Clinical Nutrition, Peking Union Medical College Hospital, PUMC, Chinese Academy of Medical Sciences, Beijing, China Correspondence to: Wei Chen. Department of Clinical Nutrition, Peking Union Medical College Hospital, PUMC, Chinese Academy of Medical Sciences, Beijing, China. Email: txchenwei@sina.com.

Submitted Mar 15, 2020. Accepted for publication Mar 25, 2021.

doi: 10.21037/hbsn-2021-9

View this article at: http://dx.doi.org/10.21037/hbsn-2021-9

The World Health Organization has suggested that people should follow these four rules of maintaining healthy eating habits, a tobacco-free lifestyle, regular physical activities, and an environment for social and emotional well-being, all of which constitutes a healthy lifestyle. Among them, a healthy diet plays the most important role. It has been well-established that healthy eating habits not only meet one's nutritional needs, but also lower one's risk of having nutrition-related chronic non-communicable diseases.

According to the "Report on Chinese Residents' Chronic Diseases and Nutrition" issued by the State Council in December 2020 (1), the chronic diseases were the leading causes of national mortality, accounting for $88.5 \%$ of the total deaths in China in 2019. Herein, the cerebrovascular diseases, cancer and chronic respiratory diseases were related to lethality, altogether accounting for $80.7 \%$ of the total mortality. What's worse, there is a tendency towards an increase in the prevalence of such chronic diseases in the Chinese people in the next few decades, mainly because of two reasons. For one thing, many Chinese people still live an unhealthy life for their imbalanced diet with excessive consumption of fat, oil, sodium, and insufficient intake of fruits, beans, and dairy products. For another thing, the rising incidence of obesity in China directly leads to increased prevalence of the chronic diseases, too. It has been showed that the rate of overweight or obesity was over $50 \%$ in adults, $19 \%$ in children under 6 and $10.4 \%$ in children or adolescents aged 6-17, respectively. In correspondence with the rise in obesity/overweight incidence, there is an increased prevalence of hypertension, diabetes, hypercholesterolemia, chronic obstructive pulmonary disease, and cancer in 2019, higher than those in 2015. To tackle with these serious issues, the "Healthy China 2030" initiative has set a comprehensive strategic plan, with implementation of crucial health-and-nutrition-related policy, including health knowledge popularization, promotion of a balanced diet as well as prevention and control of major chronic diseases (2).

Healthcare workers play a key role in practicing the "Healthy China 2030" initiative and reducing the burden of chronic diseases. However, due to the heavy workloads, stressful working environment, and irregular work schedules, many healthcare workers often sacrificed their own physical and emotional needs, in an effort to provide care for the patients. Meituan (food delivery company) has released the "Chinese Doctor Diet Report 2020", which showed that more than 7 million doctors have placed over 170 million orders for take-out foods in China, just in the first half of 2020 (3). Except for eating junk foods instead of swapping for a healthy diet, some other unhealthy behaviors also have impact on people's health, such as skipping breakfast, lacking cereals, fruits, and dairy products in the meals, and consuming too much meat and poultry. Among doctors with various background, those working in the emergency room and ICU wards had a greater tendency of being afflicted by an unhealthy diet (4).

The improper diet structure is closely correlated with the risk of developing chronic diseases. The "China Cardiovascular Health and Disease Report 2019" (5) pointed out that cardiovascular disease was ranked as the leading cause of death; however, $2 / 3$ of major coronary events and 2/5 of acute ischemic stroke could be prevented through adherence to healthy lifestyle. Through a risk attribution analysis, the 2010-2012 Chinese Nutrition and Health Survey found that (6) most cases of cardiovascular death were attributed to excessive sodium intake $(>2.0 \mathrm{~g} / \mathrm{d})(17.3 \%)$, identified as the main contributing factor. Besides, the cases were also attributed to low fruits intake $(<300.0 \mathrm{~g} / \mathrm{d})(11.5 \%)$, low omega-3 fatty acids intake $(<250.0 \mathrm{mg} / \mathrm{d})(9.7 \%)$, low nuts ingestion $(<250.0 \mathrm{mg} / \mathrm{d})$ 
$(8.2 \%)$, whole grains deficiency $(<125.0 \mathrm{~g} / \mathrm{d})(8.1 \%)$ and low consumption of vegetables $(<400.0 \mathrm{~g} / \mathrm{d})(7.3 \%)$. From 1982 to 2012, there was a transition in the diet structure of Chinese people towards reduction in grains (especially coarse grains), fresh vegetables, fruits (fewer than $50 \mathrm{~g}$ of fruits daily per person) and increased intake in animal foods and oil. A trend of reduction in salts and soy sauce ingestion was observed, though the average sodium intake is still as high as $10.5 \mathrm{~g}$ daily per person. Therefore, to improve the prevention and management of chronic diseases, there is a necessity to establish health policy which aims at government, society, and individuals, with practice of promoting the health service, enhancing nutritional education and boosting people's wellbeing in all aspects.

Western developed countries also face similar dilemma. The majority of the American population did not meet recommendations of the Dietary Guidelines for their usual dietary intake, which might be associated with the rising incidence of nutrition-related chronic diseases. The U.S. Department of Agriculture (USDA) and the Health and Human Services (HHS) update the dietary guidelines every 5 years to attempt to improve people's eating habits. The most recent guideline (2020 to 2025) provided four major principles to help people maintain health and prevent chronic diseases throughout their entire life (7): (I) following a healthy dietary habit; (II) choosing the nutrient-rich foods; (III) achieving a balanced diet comprising all kinds of food groups and limiting high-calorie foods; (IV) restricting alcohol and foods rich in added sugar, saturated fat and salt.

In order to investigate the impact of different dietary patterns on the health status of healthcare workers and find scientific evidence to effectively improve their health condition, the Clinical Nutrition Branch of the Chinese Nutrition Society conducted an online nutrition survey to measure healthcare workers' nutritional status, lifestyle, and health condition in 31 provinces, cities and municipalities in China in June 2020. In the survey, selected healthcare workers were enquired about their lifestyle, health behavior, food and nutrient intake, disease status, perception, and knowledge on health maintenance, etc. A total of 9,196 valid questionnaires were collected, with a response rate of $95.6 \%$. The data were analyzed from three perspectives (food intake and nutritional status, behaviors and health condition) and the respondents' gender and working departments were taken as stratifiers for subsequent analysis. The results showed that workers from departments of internal medicine (20\%), nutrition $(19 \%)$, obstetrics and gynecology (18\%) and surgery (12\%) had more chances of having an unhealthy diet and lifestyle than those from other medical departments. The extended and busy work shifts $(80.51 \%$ of them worked $\geq 40$ hours/ week), irregular sleep pattern with poor sleeping quality (74.78\% slept $\leq 7$ hours/day), limited access to home-cooked foods $(33.31 \%$ of them ate at home for $<7$ times/week), irregular eating behavior (30.46\%), short mealtime (34.65\% $<10$ minutes per meal), a sedentary lifestyle with daily exercise deficiency (73.74\% of them exercised for $\leq 40$ minutes/day), lack of sunlight exposure (68.07\% kept sitting for longer than 2 hours/day). All these behaviors had unfavorable impact on the health condition, with development of overweight or obesity in $32 \%$ of the healthcare workers who were investigated. Other common complaints included blurred vision or fatigue (81.32\%), being apt to catch cold (62.20\%), chronic gastroenteritis (33.05\%), constipation (31.94\%), and recurrent canker sores $(13.80 \%)$. The frequently reported chronic diseases comprised dyslipidemia (21.50\%), cardiovascular diseases $(8.42 \%)$, cancer $(5.87 \%)$, and diabetes (2.49\%). Compared to the male doctors, the females had a lower rate of diabetes, cardiovascular diseases, dyslipidemia, but a higher rate of cancer (all $\mathrm{P}<0.05$ ).

Additionally, we found that more than $50 \%$ of healthcare workers did not meet the daily nutrient intake allowance recommended by the "Chinese Dietary Guidelines (2016)" (8), with grain intake $<250 \mathrm{~g} /$ day in $59.65 \%$ of subjects, vegetable intake $<450 \mathrm{~g} /$ day in $63.41 \%$, fruit intake $<300 \mathrm{~g} /$ day in $58.88 \%$, bean intake $<10 \mathrm{~g} /$ day in $70.2 \%$, nuts intake $<10 \mathrm{~g} /$ day in $41.81 \%$, dairy products intake $<300 \mathrm{~g} /$ day in $65.95 \%$, meats intake $<100 \mathrm{~g} /$ day in $36.54 \%$, and seafood intake $<100 \mathrm{~g} /$ day in $88.75 \%$. The synthesized data also showed inadequate energy intake in the respondents and unbalanced macronutrients distribution with $46.67 \%$ of daily calories from carbohydrates and $35.37 \%$ from fats. Despite sufficient consumption of protein, iron and niacin, ingestion of many other vitamins and minerals was below the recommended dietary allowance (RDA), especially for vitamin A, vitamin C, zinc, and selenium. The healthcare workers' unhealthy eating behaviors were interpreted to be contributing factors to the unbalanced dietary ingestion. In particular, healthcare workers who ate away from home (eating at home for $<7$ times/week), ate irregularly or had short mealtime ( $<30$ minutes) had a higher risk of dietary inadequacy than those who ate regularly at home. Still, those who brought home-made meals to work were less likely to have inadequate intake of vitamin A, vitamin B2, calcium, and potassium than those who went to cafeterias or restaurants $(\mathrm{P}<0.05)$.

The finding in this cross-sectional survey about Chinese 
healthcare workers' health status and dietary habits was worrisome. Although many medical staffs in the survey had substantial self-awareness about the health issues and a willingness to maintain a healthy diet, they need to take more actions. The high-intensity workplace to some extent was not staff-friendly enough for the workers to take care of their own health, and establishing a supportive nutritional service system is of necessity. In 2020, the Chinese National Health Commission commenced the "Healthy Canteens" initiative to ensure provision of adequate nutrients to healthcare workers optimize, occupational health of them, and thereby improve the health and well-being of people all around the nation.

As for the hospital managers, awareness should be raised about the healthcare staffs' life quality and mental status, necessary policies be issued and services be provided to improve the staff's working conditions, health status and guarantee their access to a healthy and nutritious diet. On the other hand, the healthcare workers should improve their own eating habits and become more health conscious. In general, working in the fields of health science, doctors should pay attention to healthy eating themselves, which plays an important role in realizing the vision of "Healthy China 2030".

\section{Acknowledgments}

Funding: None.

\section{Footnote}

Provenance and Peer Review: This article was commissioned by the editorial office of Hepatobiliary Surgery and Nutrition. The article did not undergo external peer review.

Conflicts of Interest: The author has completed the ICMJE uniform disclosure form (available at https://hbsn. amegroups.com/article/view/10.21037/hbsn-2021-9/coif). The author has no conflicts of interest to declare.

Ethical Statement: The author is accountable for all aspects of the work in ensuring that questions related to the accuracy or integrity of any part of the work are appropriately investigated and resolved.

Open Access Statement: This is an Open Access article distributed in accordance with the Creative Commons
Attribution-NonCommercial-NoDerivs 4.0 International License (CC BY-NC-ND 4.0), which permits the noncommercial replication and distribution of the article with the strict proviso that no changes or edits are made and the original work is properly cited (including links to both the formal publication through the relevant DOI and the license). See: https://creativecommons.org/licenses/by-nc-nd/4.0/.

\section{References}

1. Report on Chinese Residents' Chronic Diseases and Nutrition (2020). Chinese State Council. December 23, 2020. Available online: http://www.gov.cn/ xinwen/2020-12/24/content_5572983.htm

2. Healthy China (2019-2030). Healthy China Action Promotion Committee. July 9, 2019. Available online: http://www.gov.cn/xinwen/2019-07/15/content_5409694. htm

3. Chinese Doctor Diet Report. Meituan. August 18, 2020. Available online: https://ishare.ifeng.com/c/s/ v002tGQzZjf9e9Shg6-_fxIMOwzQeOS8yIOYqg27-4MXZ78I

4. Zhu XR. Investigation on Dietary Habits and Nutrition Intake of Emergency ICU Medical Staffs. World Latest Med 2018;18:11-2.

5. The Writing Committee of the Report on Cardiovascular Health and Diseases in China. Interpretation of Report on Cardiovascular Health and Diseases in China 2019. Chin J Cardiovasc Med 2020;35:833-54.

6. Scientific Research Report on Dietary Guidelines for Chinese (2021). Chinese Nutrition Society 2020. Available online: https://www.cnsoc.org/learnnews/422120203.html

7. U.S. Department of Agriculture and U.S. Department of Health and Human Services. Dietary Guidelines for Americans, 2020-2025. 9th Edition. December 2020. Available online: https://www.dietaryguidelines.gov/ sites/default/files/2020-12/Dietary_Guidelines_for_ Americans_2020-2025.pdf

8. Chinese Nutrition Society. Chinese Dietary Guidelines (2016). Beijing: People's Medical Publishing House, 2016: 115-6.

Cite this article as: Chen W. Dietary health of medical workers: who's taking care of it? HepatoBiliary Surg Nutr 2021;10(2):232-234. doi: 10.21037/hbsn-2021-9 